Research Article

\title{
Analysis of genetic population structure in Acacia caven (Leguminosae, Mimosoideae), comparing one exploratory and two Bayesian-model-based methods
}

\author{
Carolina L. Pometti ${ }^{1,2}$, Cecilia F. Bessega ${ }^{1,2}$, Beatriz O. Saidman ${ }^{1,2}$ and Juan C. Vilardi ${ }^{1,2}$ \\ ${ }^{I}$ Departamento de Ecología, Genética y Evolución, Facultad de Ciencias Exactas y Naturales, \\ Universidad de Buenos Aires, Buenos Aires, Argentina. \\ ${ }^{2}$ Instituto de Ecología, Genética y Evolución de Buenos Aires, \\ Consejo Nacional de Investigaciones Cientificas y Técnicas, Buenos Aires, Argentina.
}

\begin{abstract}
Bayesian clustering as implemented in STRUCTURE or GENELAND software is widely used to form genetic groups of populations or individuals. On the other hand, in order to satisfy the need for less computer-intensive approaches, multivariate analyses are specifically devoted to extracting information from large datasets. In this paper, we report the use of a dataset of AFLP markers belonging to 15 sampling sites of Acacia caven for studying the genetic structure and comparing the consistency of three methods: STRUCTURE, GENELAND and DAPC. Of these methods, DAPC was the fastest one and showed accuracy in inferring the $K$ number of populations ( $K=12$ using the find.clusters option and $K=15$ with a priori information of populations). GENELAND in turn, provides information on the area of membership probabilities for individuals or populations in the space, when coordinates are specified $(K=12)$. STRUCTURE also inferred the number of $K$ populations and the membership probabilities of individuals based on ancestry, presenting the result $K=11$ without prior information of populations and $K=15$ using the LOCPRIOR option. Finally, in this work all three methods showed high consistency in estimating the population structure, inferring similar numbers of populations and the membership probabilities of individuals to each group, with a high correlation between each other.
\end{abstract}

Key words: Acacia caven, AFLP, GENELAND, DAPC.

Received: July 22, 2013; Accepted: October 29, 2013.

\section{Introduction}

Evaluating population genetic structure is of considerable interest because it is a precursor to addressing many other issues, such as estimating migration, identifying conservation units, and specifying phylogeographical patterns (Manel et al., 2005).

Various statistical approaches can be used to form genetic groups of populations or individuals. For statistical inferences, model-based approaches are more suitable. Bayesian clustering (Manel et al., 2005) based on HardyWeinberg and linkage equilibrium, as implemented in the STRUCTURE (Pritchard et al., 2000) or GENELAND (Guillot et al., 2005) programs, is widely used for this purpose. These programs can also consider coordinates of sampling locations. For example, when STRUCTURE is applied to population genetics, it is often useful to classify individuals of a sample into populations. In one scenario,

Send correspondence to Carolina L. Pometti. Departamento de Ecología, Genética y Evolución, Pabellón 2, Facultad de Ciencias Exactas y Naturales, Universidad de Buenos Aires, 1428 Buenos Aires, Argentina. E-mail: cpometti@ege.fcen.uba.ar. the investigator starts with a sample of individuals, aiming to determine something about the properties of populations. In a second scenario, the investigator begins with a set of predefined populations, aiming to classify individuals of unknown origin. Using the estimated allele frequencies, it is then possible to compute the likelihood of a given genotype having originated in each population. Individuals of unknown origin can be assigned to populations according to these likelihoods. Therefore, STRUCTURE uses a Bayesian clustering approach to assign individuals (probabilistically) to populations. A model is assumed in which there are $K$ populations (where $K$ may be unknown), each of which is characterized by a set of allele frequencies at each locus. This method attempts to assign individuals to populations on the basis of their genotypes, while simultaneously estimating population allele frequencies. The method can be applied to various types of markers, but it assumes that the marker loci are unlinked and in linkage equilibrium with one another within the populations. It also assumes that the populations are in Hardy-Weinberg equilibrium (Pritchard et al., 2000). In other words, the method 
assumes that any disequilibrium found is attributable to population structuration.

For cases in which the geographic locations of individuals are known and sampling is relatively even in space, spatial model-based clustering methods such as GENELAND (Guillot et al., 2005) are available to identify clusters of individuals. Assuming that populations occupy geographically delimited areas, the use of spatial information increases the power of correctly detecting the underlying population structure (Bonin et al., 2007). The statistical model implemented in GENELAND helps inferring and locating genetic discontinuities between populations in space from individual multilocus genetic data. The central assumption is that some spatial dependence is often present among individuals. Based on this sensible assumption, a hierarchical spatial model was developed in which a priori information on how the individuals are spatially organized is formally injected. In addition to detecting genetic discontinuities between populations, the method also addresses other points, such as denoising blurred coordinates of sampled individuals, estimating the number of populations in the studied area, quantifying the amount of spatial dependence in the data, assigning individuals to their population of origin, and detecting individual migrants between populations (Guillot et al., 2005).

One of the shortcomings of Bayesian clustering methods is related with the assumption of Hardy-Weinberg and linkage equilibrium within populations. However, in many cases, this assumption is not tenable. A technical yet critical limitation is the considerable computation time required for analyzing large datasets. In order to satisfy the need for less computer-intensive approaches, multivariate analyses seem particularly appealing, as they are specifically devoted to extracting information from large datasets. This is how the Discriminant Analysis of Principal Components (DAPC) was developed. DAPC is based on data transformation, using principal components analysis (PCA) as a prior step to discriminant analysis (DA), which ensures that variables submitted to DA are perfectly uncorrelated, and that their number is less than that of the analyzed individuals. Without necessarily implying a loss of genetic information, this transformation allows DA to be applied to any genetic data. Two options for DAPC are offered, depending on whether group priors are known or not (Jombart et al., 2010).

In this context, since plant populations are not randomly arranged assemblages of genotypes, but are structured in space and time, the above mentioned programs allow a fine-scale study of the genetic structure of these populations. This genetic structure may be manifested among geographically distinct populations, within a local group of plants, or even in the progeny of individuals. Ecologic factors affecting reproduction and dispersal are likely to be particularly important in determining genetic structure. Also, spatial and genetic patterns are often assumed to result from environmental heterogeneity and differential selection pressures (Loveless and Hamrick, 1984).

In this paper, we describe a study on natural Argentinean populations of the plant species Acacia caven (Leguminosae, Mimosoideae). This species is an extremely wide-ranging one that probably originated in the warm temperate to subtropical biogeographic region known as the Gran Chaco of southern South America, due to its great morphologic diversity. This small legume species is found in six countries and is considered to have certain potential as a managed silvopastoral crop (Aronson and Ovalle, 1989). Fruit size and shape are highly variable in A. caven. In 1992, Aronson recognized six varieties for this species, including $A$. caven var. caven, A. caven var. dehiscens, $A$. caven var. sphaerocarpa, $A$. caven var. stenocarpa, $A$. caven var. microcarpa and A.caven var. macrocarpa, based on both morphologic traits (Aronson 1992; Pometti et al., 2007) and molecular markers (Pometti et al., 2010). Argentina is the only country where all varieties cohabit (Aronson, 1992).

In this context, the main objective of the present work was to study the genetic structure of 15 populations of the six varieties of Acacia caven, using a dataset of AFLP markers. To accomplish this objective, we used two model-based approaches (STRUCTURE and GENELAND) and the exploratory method DAPC for estimating genetic structure and compared the consistency of the three methods.

\section{Materials and Methods}

\section{Description of the dataset}

In this study, a real dataset was used to compare the results of genetic structure analyses made by alternative approaches. This dataset consists of AFLP patterns of 224 individuals of the six varieties of Acacia caven (Leguminosae, Mimosoideae), collected from 15 sampling sites (Table 1). The distances between the sampling sites are shown in Table 2.

The AFLP assay was performed as described by Vos et al. (1995), with a slight modification, as described in Pometti et al. (2012). This technique was used to investigate genetic variation within and among natural populations of $A$. caven from five eco-regions: Wet Chaco, Dry Chaco, Espinal, Pampa and Puna (Burkart et al., 1999). From the individuals studied by means of AFLP markers, 225 bands were obtained. Each AFLP band was considered as a single biallelic locus with one amplifiable and one null allele. Bands with the same migration distance were considered homologous. Data were scored manually as band presence (1) or absence (0).

\section{Methods to assess population structure}

As mentioned before, different approaches were used here to identify spatial structure in $A$. caven populations: 
Table 1 - Populations of Acacia caven sampled in this study.

\begin{tabular}{|c|c|c|c|c|c|c|}
\hline Variety & Eco-region & Population & Population code & Latitude $\left({ }^{\circ} \mathrm{S}\right)$ & Longitude $\left({ }^{\circ} \mathrm{W}\right)$ & $\begin{array}{c}\text { Number of individuals } \\
\text { analyzed }\end{array}$ \\
\hline A. caven var caven & Pampa & Costanera Sur & $\mathrm{CS}$ & $34^{\circ} 38^{\prime} 10.71^{\prime \prime}$ & $58^{\circ} 42^{\prime} 44.08^{\prime \prime}$ & 14 \\
\hline A. caven var caven & Pampa & Gualeguaychú & GY & $33^{\circ} 22^{\prime} 4.00^{\prime \prime}$ & $58^{\circ} 44^{\prime} 3.00^{\prime \prime}$ & 22 \\
\hline A. caven var caven & Puna & Coiruro & $\mathrm{CI}$ & $23^{\circ} 53^{\prime} 34.00^{\prime \prime}$ & $65^{\circ} 27^{\prime} 30.00^{\prime \prime}$ & 18 \\
\hline A. caven var caven & Puna & Campo Quijano & CQ & $24^{\circ} 55^{\prime} 12.00^{\prime \prime}$ & $65^{\circ} 39^{\prime} 0.00^{\prime \prime}$ & 13 \\
\hline A. caven var caven & Puna & Ruta Nueve & $\mathrm{RN}$ & $24^{\circ} 39^{\prime} 48.00^{\prime \prime}$ & $65^{\circ} 22^{\prime} 49.00^{\prime \prime}$ & 14 \\
\hline A. caven var macrocarpa & Puna & El Carril & $\mathrm{EC}$ & $25^{\circ} 4^{\prime} 58.80^{\prime \prime}$ & $65^{\circ} 28^{\prime} 1.20^{\prime \prime}$ & 16 \\
\hline A. caven var macrocarpa & Puna & Tolombón & TO & $26^{\circ} 11^{\prime} 8.00^{\prime \prime}$ & $65^{\circ} 56^{\prime} 7.00^{\prime \prime}$ & 14 \\
\hline A. caven var microcarpa & Wet Chaco & Vivero Forestal & $\mathrm{VF}$ & $26^{\circ} 16^{\prime} 0.00^{\prime \prime}$ & $58^{\circ} 17^{\prime} 41.64^{\prime \prime}$ & 12 \\
\hline A. caven var stenocarpa & Wet Chaco & Formosa & FS & $26^{\circ} 16^{\prime} 13.20^{\prime \prime}$ & $58^{\circ} 17^{\prime} 7.92^{\prime \prime}$ & 12 \\
\hline A. caven var stenocarpa & Wet Chaco & YPF & YP & $26^{\circ} 11^{\prime} 26.76^{\prime \prime}$ & $58^{\circ} 9^{\prime} 23.82^{\prime \prime}$ & 12 \\
\hline A. caven var sphaerocarpa & Espinal & Iberá & IB & $28^{\circ} 15^{\prime} 40.13^{\prime \prime}$ & $56^{\circ} 30^{\prime} 20.38^{\prime \prime}$ & 18 \\
\hline A. caven var dehiscens & Dry Chaco & Las Gemelas & LG & $30^{\circ} 53^{\prime} 26.10^{\prime \prime}$ & $64^{\circ} 30^{\prime} 13.50^{\prime \prime}$ & 14 \\
\hline A. caven var dehiscens & Dry Chaco & Pan de Azúcar & PA & $31^{\circ} 15^{\prime} 58.90^{\prime \prime}$ & $64^{\circ} 20^{\prime} 28.60^{\prime \prime}$ & 12 \\
\hline A. caven var dehiscens & Dry Chaco & Vaquerías & VA & $31^{\circ} 23^{\prime} 38.93^{\prime \prime}$ & $63^{\circ} 51^{\prime} 30.87^{\prime \prime}$ & 12 \\
\hline A. caven var dehiscens & Dry Chaco & Valle Hermoso & VH & $31^{\circ} 7^{\prime} 1.20^{\prime \prime}$ & $64^{\circ} 28^{\prime} 58.80^{\prime \prime}$ & 21 \\
\hline
\end{tabular}

Table 2 - Pairwise geographic distances in kilometers between Acacia caven sampling sites.

\begin{tabular}{|c|c|c|c|c|c|c|c|c|c|c|c|c|c|c|}
\hline Pop & CQ & $\mathrm{CS}$ & $\mathrm{EC}$ & FS & GY & IB & LG & PA & $\mathrm{RN}$ & TO & VA & VF & $\mathrm{VH}$ & YP \\
\hline CI & 111.00 & 1348.53 & 123.80 & 768.28 & 1226.00 & 1017.75 & 809.44 & 832.00 & 79.21 & 263.14 & 833.53 & 771.49 & 856.92 & 780.57 \\
\hline CQ & & 1280.00 & 25.00 & 752.46 & 1166.50 & 982.49 & 700.00 & 749.50 & 39.28 & 159.26 & 744.23 & 751.00 & 750.71 & 764.50 \\
\hline CS & & & 1238.29 & 910.82 & 185.00 & 745.31 & 655.00 & 645.00 & 1273.21 & 1155.11 & 650.00 & 911.38 & 664.42 & 932.57 \\
\hline EC & & & & 730.82 & 1121.00 & 961.09 & 674.07 & 716.00 & 50.33 & 139.09 & 701.21 & 729.25 & 683.00 & 743.00 \\
\hline FS & & & & & 777.15 & 282.15 & 779.31 & 801.41 & 731.84 & 759.85 & 780.43 & 3.00 & 800.00 & 15.80 \\
\hline GY & & & & & & 617.17 & 584.38 & 574.00 & 1198.25 & 1046.00 & 593.30 & 770.79 & 593.50 & 793.76 \\
\hline IB & & & & & & & 832.84 & 827.86 & 976.03 & 964.59 & 794.48 & 283.43 & 838.96 & 282.15 \\
\hline LG & & & & & & & & 43.50 & 738.31 & 547.38 & 29.40 & 783.78 & 35.20 & 809.13 \\
\hline PA & & & & & & & & & 798.50 & 576.00 & 21.00 & 795.39 & 22.44 & 823.54 \\
\hline $\mathrm{RN}$ & & & & & & & & & & 194.06 & 767.15 & 730.38 & 727.22 & 745.00 \\
\hline TO & & & & & & & & & & & 593.13 & 759.56 & 565.74 & 774.56 \\
\hline VA & & & & & & & & & & & & 775.78 & 4.00 & 797.27 \\
\hline VF & & & & & & & & & & & & & 799.00 & 16.41 \\
\hline VH & & & & & & & & & & & & & & 824.37 \\
\hline
\end{tabular}

two Bayesian-model-based and one exploratory method. The first one was the spatial cluster model implemented in the GENELAND package (Guillot et al., 2005) of the R program (R Development Core Team, 2011). Different sets of parameters (MCMC, thinning and burn-in) were used in different test runs, in order to find the optimal parameters by the time taken for the run. Finally, following the recommendation of the user's manual, the Markov chain Monte Carlo (MCMC) repetitions were set at 100,000, thinning was set at 100, and the burn-in period was set at 200 (we eliminated the first 200 iterations whenever the curve was not constant); the number of groups $(K)$ to be tested was set at 1-15. All individuals were assigned to $K$ populations $(1 \leq K \leq 15)$ based on their multilocus genotype and the spatial coordinates. To ensure that the run was long enough, we obtained 10 different runs and compared the parameter estimates ( $K$, individual population membership, maps). The best result was chosen, based on the highest average posterior probability. The other Bayesian-model-based cluster analysis was performed using the STRUCTURE program version 2.3.3 (Pritchard et al., 2009). This analysis was performed twice: once without prior information of the populations to which the individuals belonged, and once with prior information on the populations (LOCPRIOR model). 
In both cases, the burn-in period and the number of $\mathrm{MCMC}$ repetitions were set, respectively, at 50,000 and 100,000. An admixture model was used, with correlated allele frequencies. $K$ was set at $1-15$, and the highest $K$ value was identified as the run with the highest likelihood value, as recommended by Pritchard et al. (2000). In addition, $K$ values were averaged across 10 iterations. The exploratory Discriminant Analysis of Principal Components (DAPC) was applied, using the adegenet package (Jombart, 2008) (function dapc) for software R (R Development Core Team, 2011). This analysis was also performed both with and without prior information on individual populations. Whenever group priors were unknown, the number of clusters was assessed using the find.clusters function, which runs successive $K$-means clustering with increasing number of clusters (k). For selecting the optimal number of clusters, we applied the Bayesian Information Criterion (BIC) for assessing the best supported model, and therefore the number and nature of clusters, as recommended by Jombart el al. (2010).

\section{Comparison of individual groupings in the different methods}

The probabilities of posterior population membership of individuals obtained by all grouping methods used were converted into between-individual Euclidean distances. Pairwise comparisons of these distance matrices were performed by means of the Mantel test using the ade 4 package of R (Chessel et al., 2004).

\section{Results}

Analysis of the Acacia caven AFLP dataset obtained using GENELAND yielded a modal number of populations of 12, varying from 11-13 in different runs (Table 3). The run with the highest average posterior probability was cho-

Table 3 - Multiple runs for inferring the number of populations using GENELAND software.

\begin{tabular}{lccc}
\hline Run & Modal number & \% of modal number & $\begin{array}{c}\text { Mean of probability } \\
\text { density }\end{array}$ \\
\hline 1 & 12 & 37.20 & -62443.26 \\
2 & 12 & 37.80 & -60538.68 \\
3 & 11 & 38.90 & -60964.61 \\
4 & 13 & 32.80 & -60583.12 \\
5 & 11 & 36.80 & -61215.83 \\
6 & 13 & 33.40 & -60874.19 \\
7 & 12 & 36.40 & -60953.19 \\
$\mathbf{8}$ & $\mathbf{1 2}$ & $\mathbf{3 6 . 2 0}$ & $\mathbf{- 5 9 9 9 9 . 6 6}$ \\
9 & 12 & 36.80 & -61164.86 \\
10 & 12 & 36.00 & -60860.80 \\
\hline
\end{tabular}

In bold: highest average posterior probability. sen to base the conclusions on. The number of populations simulated from posterior distribution (Figure 1) displays a clear mode at $K=12$. MCMC clearly converges within the first 10,000 iterations (Figure 1). Two populations, VA and PA (belonging to var. dehiscens), were included in one of the groups produced by GENELAND (Figure 2, row 3, column 2), and the other group identified comprises the VF, FS, and YP populations (belonging to vars. microcarpa and stenocarpa) (Figure 2, row 2, column 2). In both cases, the populations grouped together are geographically very close to each other. Each of the remaining groups corresponds to a single sampling site. The comparison of posterior probability of assignment of individuals to populations led to unequivocal results, assigning each individual to the population to which it belongs, except for those previously mentioned individuals that are in the same group of populations ( $100 \%$ of correct assignation).

Data analysis using STRUCTURE with no prior distribution specified revealed that $K=11$ had the highest mean probability of density value $(\operatorname{Ln} P(D)=-16832.60)$, after which this value plateaus, suggesting that the optimal number of $K$ was 11. In this analysis (Figure 3a), individuals of populations FS, VF, and YP are grouped together, the same occurs with individuals of populations PA and VA, and a third group joins together individuals of populations $\mathrm{CQ}$ and RN that belong to the var. caven and are both located in the Puna eco-region (Figure 3a). The assignation of individuals to populations was $96.4 \%$ correct.

When the LOCPRIOR option was used, $K=15$ had the highest mean probability of density value $(\operatorname{Ln} P(D)=$ -17065.30), suggesting that each population corresponded to a single sampling site (Figure $3 \mathrm{~b}$ ). Moreover, the STRUCTURE results detected admixture of individuals in all populations with both models (Figure $3 \mathrm{a}, \mathrm{b}$ ). The assignation of individuals to populations was $94.2 \%$ correct.

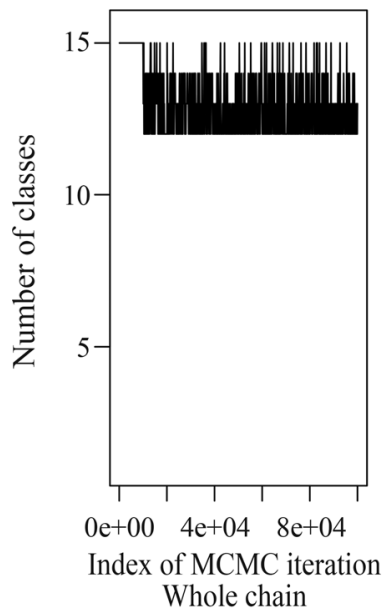

Number of populations along the chain after burnin

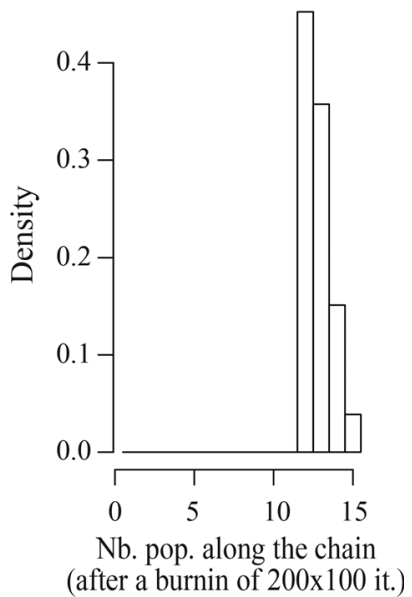

Figure 1 - Plot of the number of populations simulated from the posterior distribution obtained with GENELAND. 

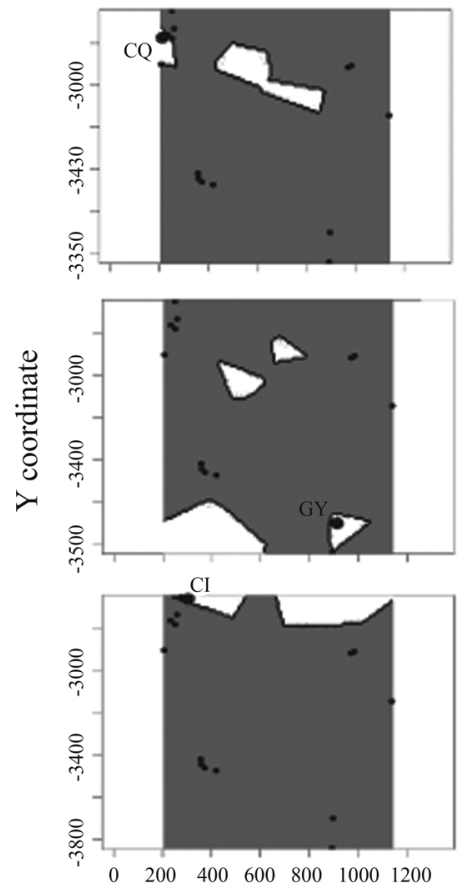
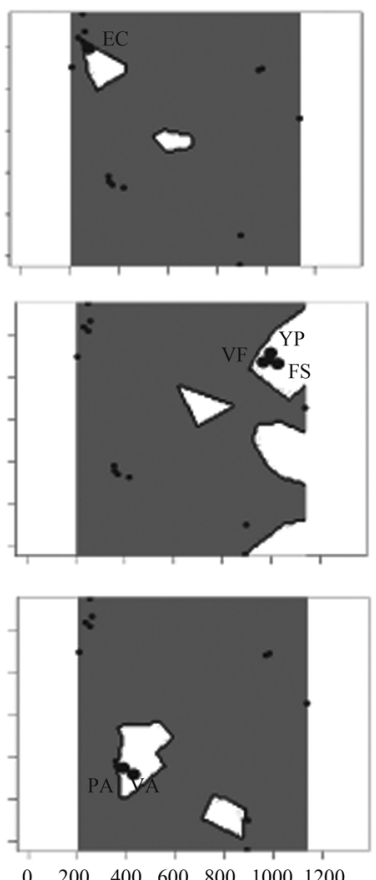
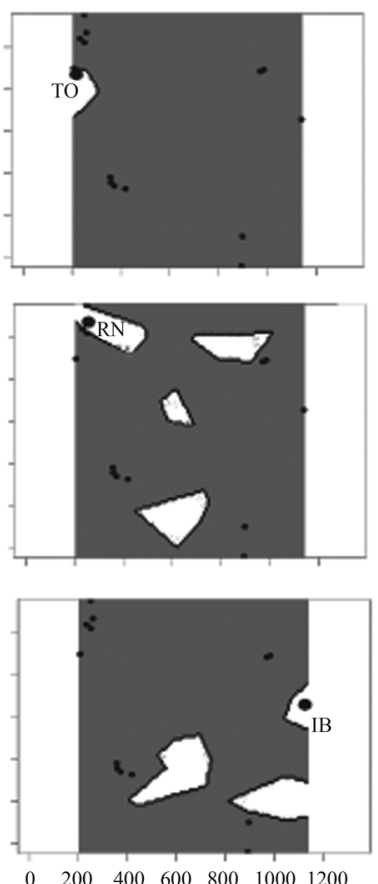

$\begin{array}{lllllll}0 & 200 & 400 & 600 & 800 & 1000 & 1200\end{array}$
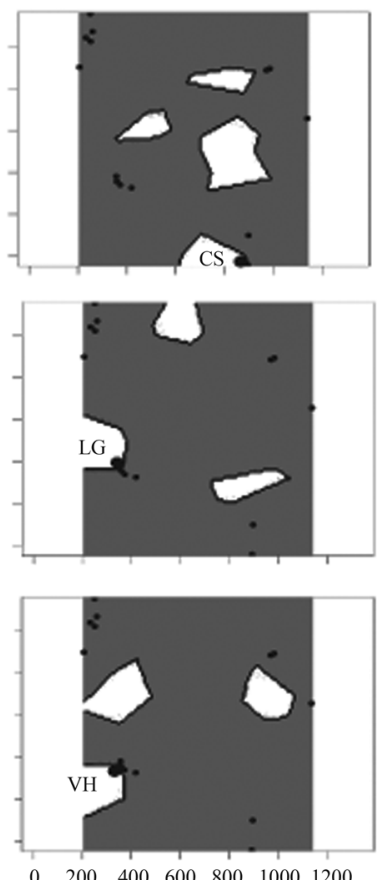

Figure 2 - Spatial distribution of each group defined by GENELAND at $K=12$. Population codes are given in Table 1 .

a)

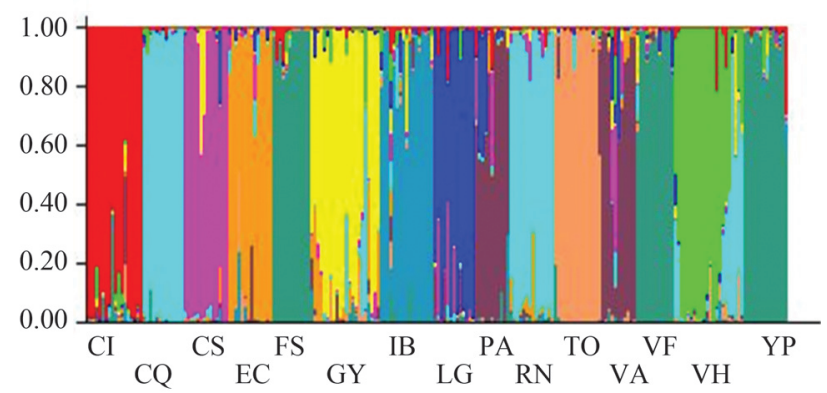

b)

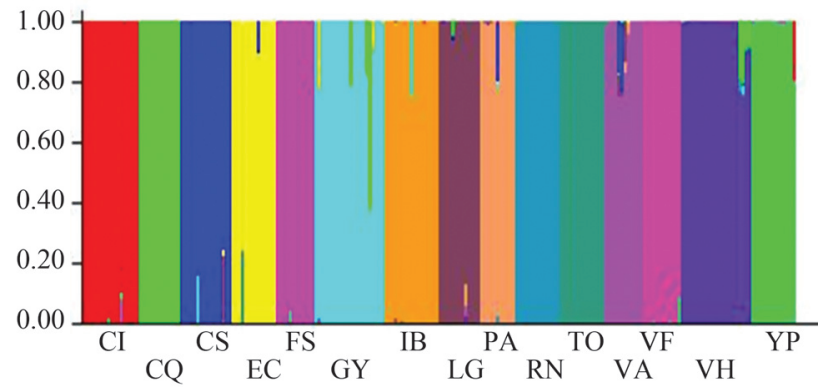

Figure 3 - Clustering of individuals by STRUCTURE. Each individual is represented by a vertical bar that is partitioned into colored segments that represent the individual's estimated membership fractions. Same color in different individuals indicates that they belong to the same cluster. a) $K=11$, estimated with no prior distribution of populations; b) $K=15$, estimated with LOCPRIOR option. Population codes are given in Table 1.

DAPC analysis was first made without any a priori group assignment. To obtain the optimal number of clusters with the find.clusters function, 70 axes that represented more than $88 \%$ of the total variance were retained. The program covered a range of possible clusters from 1 to 15 . The lowest BIC value (1137.35) corresponded to $K=12$. For DAPC analysis, 70 PCA axes and three discriminant functions were retained ( $52.3 \%$ of variance). One of the clusters included individuals of populations VF, FS, and YP, a second cluster joined PA and VA, and the remaining clusters were rather consistent with the rest of the sampling sites. The scatterplot of individuals on the two principal components of DAPC (Figure 4a) showed that the 12 clusters formed four groups. The consistency between prior and posterior assignment was $84.8 \%$.

In the second analysis, the clusters were defined a priori, according to the sampling site. Also in this case, 70 axes of the PCA were retained for DAPC, corresponding to more than $88.8 \%$ of the variance, and three discriminant functions were obtained $(53.9 \%$ of the variance). The scatterplot shows overlapping between the a priori defined groups (Figure 4b); the consistency between prior and posterior assignment was $88.8 \%$.

The results obtained from the two approaches can also be compared with the posterior probability plots corresponding to the groups defined by the find.clusters procedure (Figure 5a) and with the groups defined by the sampling site (Figure 5b).

Regarding the consistency between prior and posterior assignment of individuals to groups (Table 4), the maximum corresponded to GENELAND (100\%), whereas the lowest consistency was obtained by DAPC without information on population membership (84.8\%). Pairwise com- 

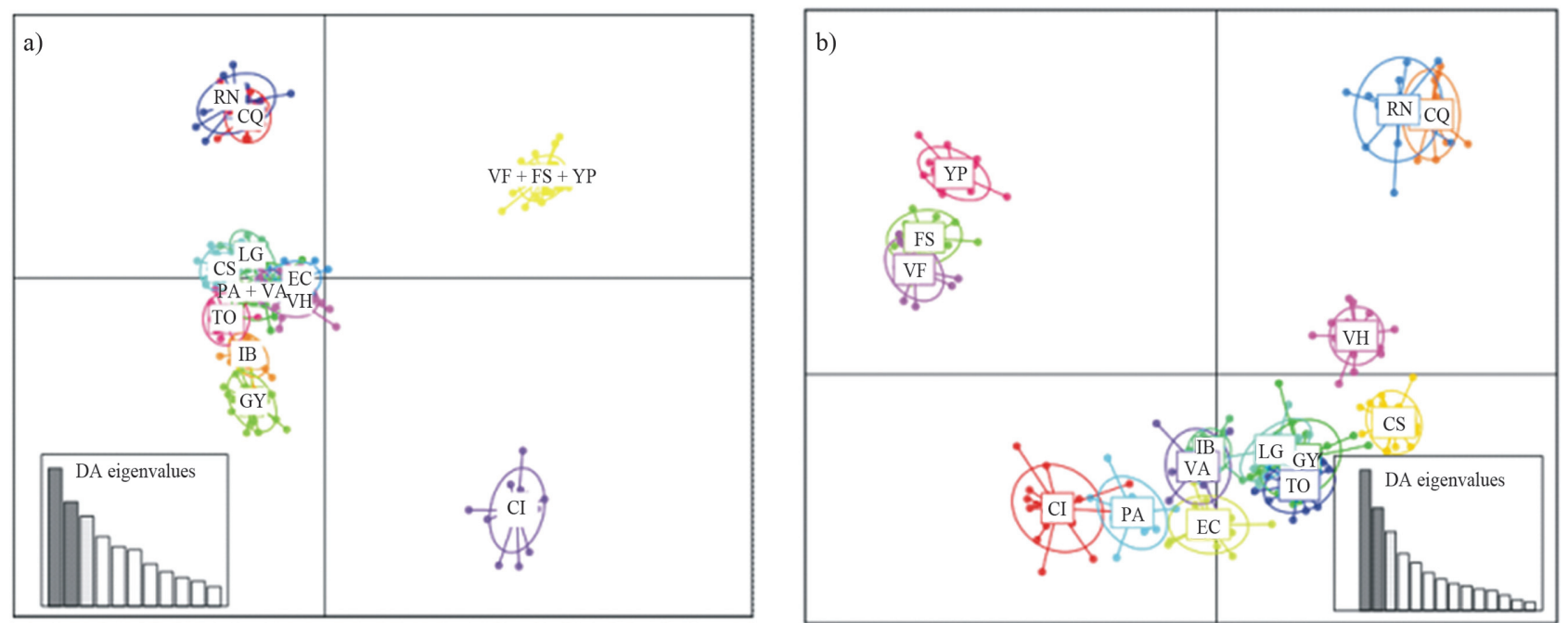

Figure 4 - Scatterplot of individuals on the two principal components of DAPC. The graph represents the individuals as dots and the groups as inertia ellipses. Eigenvalues of the analysis are displayed in inset: a) obtained with the find.clusters option, b) with clusters defined a priori according to the sampling site. Population codes are given in Table 1.
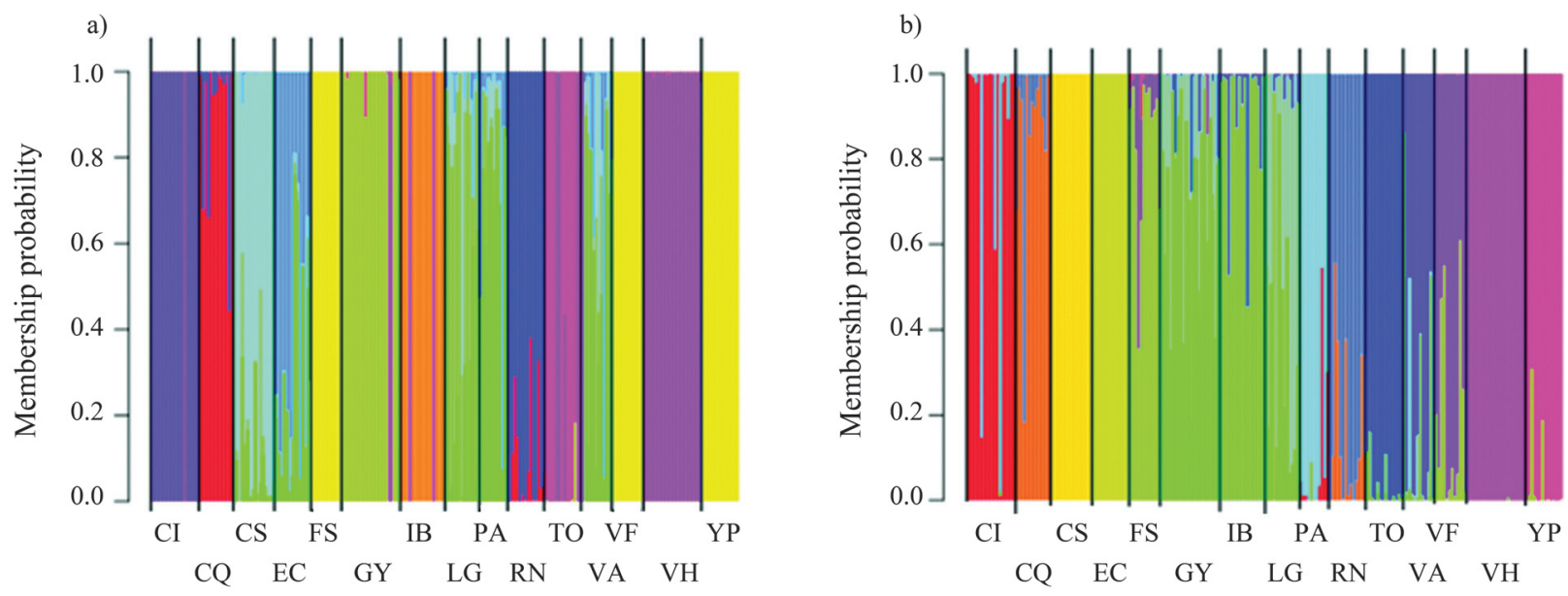

Figure 5 - STRUCTURE-like plot of DAPC analysis for a global picture of the clusters composition. Each individual is represented by a vertical colored line. Same color in different individuals indicates that they belong to the same cluster. a) $K=12$, obtained with find.clusters option; b) $K=15$, obtained with a priori information of sampling sites. Population Codes are given in Table 1.

parison of distances between individuals obtained from the probabilities of posterior assignment of population membership of individuals resulting from all five grouping methods (Table 4) revealed highly significant correlations ( $\mathrm{p}<0.0005$, based on 2000 permutations) in all cases. The highest consistency value $(r=0.811)$ corresponded to the groupings obtained by GENELAND and STRUCTURE for the admixture model without prior information on population membership. The grouping obtained by DAPC without prior information on population membership showed the lowest correlation estimates when compared with most of the other grouping methods.

\section{Discussion}

The analysis of genetic diversity within species is vital for understanding the evolutionary processes, both at the population and at the genomic levels. Several statistical packages recently developed which offer a panel of standard as well as more sophisticated analyses have been reviewed by Excoffier and Heckel (2006). Most data analyses require the use of more than one program and should start with generalist packages to uncover the basic properties of the data, followed by the use of specialized methodologies to address more specific questions (Excoffier and Heckel, 2006).

In line with this recommendation, we evaluated the consistency of different methodological approaches for analyzing genetic properties of Acacia caven populations, a shrub widely distributed in South America. This species plays an important role in arid ecosystems, as it contributes to the fixation of atmospheric nitrogen, provides fruits and leaves to herbivores, and stabilizes soils by fixing dunes. In 
Table 4 - Pairwise comparison of distances between individuals obtained from the probabilities of posterior population membership of individuals, obtained by all five grouping methods. $K=$ number of clusters; $r=$ correlation coefficient; $\mathrm{p}<0.0005$; STR $1=$ STRUCTURE analysis without prior information; STR 2 = STRUCTURE analysis with LOCPRIOR option; DAPC 1= DAPC analysis with find.clusters option; DAPC $2=$ DAPC analysis with a priori information of populations.

\begin{tabular}{|c|c|c|c|c|c|c|c|}
\hline & \multicolumn{7}{|c|}{$r$} \\
\hline & $K$ & $\%$ of correct assignment & GENELAN D & STR 1 & STR 2 & DAPC 1 & DAPC 2 \\
\hline Sampling sites & 15 & - & & & & & \\
\hline GENELAND & 12 & 100 & - & & & & \\
\hline STR 1 & 11 & 96.4 & 0.811 & - & & & \\
\hline STR 2 & 15 & & 0.726 & 0.710 & - & & \\
\hline DAPC 1 & 12 & 84.8 & 0.612 & 0.616 & 0.577 & - & \\
\hline DAPC 2 & 15 & 88.8 & 0.769 & 0.673 & 0.716 & 0.607 & - \\
\hline
\end{tabular}

addition, it is an appreciated natural resource for local settlers, because it provides fire wood, charcoal and forage for livestock. Due to its great plasticity, it is used in the reforestation of degraded ecosystems (Karlin et al., 1997).

In this work, we chose one exploratory and two Bayesian-model-based methods to infer the genetic structure of $A$. caven species from 15 sampling sites. The exploratory method used here was DAPC that seeks synthetic variables, the discriminant functions, which show differences between groups as best as possible, while minimizing variation within clusters (Jombart, 2012). Using the find.clusters option in this analysis, the number of populations inferred was $K=12$, grouping together $\mathrm{VF}, \mathrm{FS}$, and YP and also PA and VA. DAPC analysis is preferred when groups are often unknown or uncertain and there is a need for identifying genetic clusters before describing them. In this work, we found that those sampling sites that grouped together in the same cluster were the geographically closer ones. When we defined the prior groups for the DAPC analysis, the inferred $K$ was 15 , the same as the number of sampling sites. In both cases, the percentage of variance explained by the three discriminant functions was $<54 \%$. This could be attributed to the reduction of variables achieved by DAPC; in other words, we had 225 loci or variables, and this method reduced (in this case) the number of composed variables to the 70 more informative axes.

Additionally, two Bayesian analyses were applied to the data to study the genetic structure of the samples (GENELAND and STRUCTURE). When STRUCTURE was run with the LOCPRIOR option, the $K$ estimated was coincident with the number of data sampling sites $(K=15)$. When using STRUCTURE, it is usually assumed that all partitions of individuals are a priori approximately equally likely. Since the number of possible partitions is immense, it takes highly informative data for STRUCTURE to conclude that any particular partition of individuals into clusters has compelling statistical support. In contrast, the LOCPRIOR models assume that, in practice, individuals from the same sampling location often come from the same population. Therefore, the LOCPRIOR models are set up to expect that the sampling locations may be informative about ancestry. If the data suggest that the locations are informative, then the LOCPRIOR models allow STRUCTURE to use this information (Pritchard et al., 2010).

GENELAND analysis in turn showed that the $15 \mathrm{~A}$. caven populations studied could be grouped into $K=12$ independent groups, indicating that each sampling site represented a single Mendelian population, with the exception of VA and PA, and FS, YP, and VF, which would correspond to two clusters. STRUCTURE analysis without prior information of populations showed that the optimal number of populations was $K=11$, joining together populations CQ and $\mathrm{RN}$. The other 10 groups constituted were coincident with those detected by GENELAND. The slight difference between analyses regarding the detection of the number of $K$ could be attributed to the model chosen, since GENELAND was run with previous information of geographic coordinates, tending to favor partitions that are spatially organized, while STRUCTURE was not. Similar differences in behavior between GENELAND and STRUCTURE were noted by Guillot et al. (2005) when comparing the dataset of Montana wolverines (Gulo gulo) recorded by Cegelski et al. (2003), as STRUCTURE inferred $K=3$, whereas GENELAND inferred $K=4$. In our case, GENELAND grouped together $A$. caven populations that were geographically and genetically closer and located in the same eco-region, such as VA and PA, and FS, VF, and YP. On the other hand, STRUCTURE detected the genetically similar groups. Variety caven is the most widespread (a generalist, in terms of ecology range), and here we analyzed five of its populations from two eco-regions. One could expect to find these populations grouped together according to the eco-region and the variety they belong to. However, the results of the Puna eco-region suggest that there the populations are less connected to each other by gene flow than the populations of the other ecoregions, since $\mathrm{CI}$ was not grouped together with $\mathrm{CQ}$ and $\mathrm{RN}$ in the STRUCTURE analysis. A possible explanation for this clustering could be that the geographic distances be- 
tween CQ and RN were smaller than that from CI, and the genetic and geographic distances among the populations studied here have shown to be significantly correlated (Pometti et al., 2012). Moreover, although these three populations belong to the same variety and the same eco-region, they were found at different altitudes: $\mathrm{RN}$ at $1305 \mathrm{~m}$ o.s.l., CQ at $1511 \mathrm{~m}$ o.s.1., and CI at $2089 \mathrm{~m}$ o.s.l. This results in an environment of patchy vegetation, because of the presence of mountains that separate $\mathrm{CI}$ from RN and CQ. It has been well documented that marginal populations are often less variable than populations within the primary range (Blows and Hoffmann, 1993; Deng et al., 2009). The results obtained for the variety caven from the Puna ecoregion could be explained by the observations of Hamrick and Godt (1990) and Maguire et al. (2000) that populations located at range margins are more isolated from sources of immigrants and are thus more prone to genetic bottlenecks.

When comparing the number $K$ of populations estimated in the three methods, DAPC using the find.clusters option proved as accurate in detecting population clusters as STRUCTURE without prior information of populations and GENELAND.

When prior groups were defined, the DAPC results were coincident with those obtained by STRUCTURE with the LOCPRIOR option, where $K=15$. As previously explained, in both cases the sampling locations were informative about ancestry.

A significant degree of genetic differentiation among A. caven populations was observed using the three methods, since $K$ ranged from 11 to 15 , showing a high level of structuration in the 15 sampling sites studied. The most evident associations among populations were found for PA and VA, and FS, VF and YP in all analyses, and for CQ and RN with STRUCTURE. No other association between populations by eco-region or variety was observed consistently with the tree methods used.

The three methods used here to infer population structure also provide coefficients of membership probabilities of each individual to the different groups, based on the retained discriminant functions in the case of DAPC, or based on ancestry in the case of STRUCTURE and GENELAND. While DAPC coefficients are different from the admixture coefficients of softwares like STRUCTURE or GENELAND, they can still be interpreted as proximities of individuals to the different clusters. Membership probabilities also provide indications of how clear-cut genetic clusters are (Jombart, 2012). The highest membership probabilities of each individual for the different groups were obtained by GENELAND, followed by STRUCTURE with prior definition of groups, STRUCTURE without population information, DAPC with prior definition of groups, and the lowest membership probabilities were those observed by DAPC without information on population membership. This means that the three methods and their variants provided accurate assign- ments of individuals, ranging from $84.8 \%$ for DAPC using the find.clusters option to $100 \%$ for GENELAND.

In conclusion, of the three methods used here, DAPC proved to be the fastest one, showing accuracy in inferring the $K$ number of populations and the membership probabilities of each individual for the different groups in a short computational time (only a few minutes, while STRUCTURE and GENELAND needed four or five days to perform the analysis). So, DAPC should be preferred as a starting point when working with large datasets and several sampling sites, as recommended by Excoffier and Heckel (2006). GENELAND, on the other hand, provides information on the area of membership probabilities for individuals or populations in space, when coordinates are specified; moreover, the number of population units is treated as an unknown parameter (Guillot et al., 2005). STRUCTURE, in addition to inferring the number of $K$ populations and the membership probabilities of individuals based on ancestry, allows a hierarchical analysis of sampling sites from $K=2$ to $K=n$, where $n$ is the number of populations estimated with the highest mean probability of density value (Tishkoff et al., 2009; Pometti et al., 2012). The two latter analyses present the disadvantage of being more time-consuming and relying on assumptions, such as the type of population subdivision and Hardy-Weinberg and linkage equilibrium inside populations. Finally, in this work, all three methods showed high consistency in estimating the population structure of $A$. caven, inferring similar numbers of populations and membership probabilities of individuals to each group, with a high correlation between each other. This consistency may be interpreted in a similar way as the consistency between phenetic and cladistic analyses, which, although being based on different assumptions, reveal in many cases similar associations between phylogenetically related groups.

\section{Acknowledgments}

This work was supported by grants from the Universidad de Buenos Aires (EX201 and 20020100100008 to B.O.S.) and the Consejo Nacional de Investigaciones Científicas y Técnicas (PIP 11220090100147 to J.C.V.).

\section{References}

Aronson J (1992) Evolutionary Biology of Acacia caven (Leguminosae, Mimosoideae): Infraspecific variation in fruit and seed characters. Ann Mo Bot Gard 79:958-968.

Aronson J and Ovalle C (1989) Report on a study of the natural variability, biogeography and potential for genetic improvement of Acacia caven. Bull Int Group Study Mimosoideae 17:111-121.

Blows MW and Hoffmann AA (1993) The genetics of central and marginal populations of Drosophila serrata. I. Genetic Variation for Stress Resistance and Species Borders. Evolution 47:1255-1270. 
Bonin A, Ehrich D and Manel S (2007) Statistical analysis of amplified fragment length polymorphism data: A toolbox for molecular ecologists and evolutionists. Mol Ecol 16:37373758.

Burkart R, Bárbaro NO, Sánchez RO and Gómez DA (1999) Ecorregiones de la Argentina. Administración de Parques Nacionales, Buenos Aires, 43 p.

Cegelski C, Waits L and Anderson J (2003) Assessing population structure and gene flow in Montana wolverines (Gulo, gulo) using assignment-based approaches. Mol Ecol 12:29072918.

Chessel D, Dufour AB and Thioulouse J (2004) The ade4 package-I- One-table methods. R News 4:5-10.

Deng S, Huang Y, He H, Tan F, Ni X, Jayatissa LP, Hettiarachi S and Shi S (2009) Genetic diversity of Aegiceras corniculatum (Myrsinaceae) revealed by amplified fragment length polymorphism (AFLP). Aquat Bot 90:275-281.

Excoffier L and Heckel G (2006) Computer programs for population genetics data analysis: A survival guide. Nat Rev Genet 7:745-758

Guillot G, Mortier F and Estoup A (2005) GENELAND: A computer package for landscape genetics. Mol Ecol Notes 5:712-715.

Hamrick JC and Godt JW (1990) Allozyme diversity in plant species. In Brown HD, Clegg MT, Kahler AL and Weir BS (eds.) Plant Population Genetics, Breeding, and Genetic Resources. Sinauer Associates, Sunderland, p 43-65.

Jombart T (2008) Adegenet: A R package for the multivariate analysis of genetic markers. Bioinformatics 24:1403-1405.

Jombart T, Devillard S and Balloux, F (2010) Discriminant analysis of principal components: A new method for the analysis of genetically structured populations. BMC Genetics 11:94.

Karlin OU, Coirini RO, Catalan L and Zapata R (1997) Acacia caven. In: Oficina Regional de la FAO para America Latina y el Caribe (ed) Especies Arbóreas y Arbustivas para las Zonas Áridas y Semiáridas de América Latina, Zonas Áridas y Semiáridas. FAO/PNUMA, Santiago, pp 157-167.

Loveless MD and Hamrick JL (1984) Ecological determinants of genetic structure in plant populations. Ann Rev Ecol Syst 15:65-95

Maguire TL, Saenger P, Baverstock P and Henry R (2000) Microsatellite analysis of genetic structure in the mangrove species Avicennia marina (Forsk) Vierh. (Avicenniaceae). Mol Ecol 9:1853-1862.
Manel S, Gaggiotti O and Waples RS (2005) Assignment methods: Matching biological questions with appropriate techniques. Trends Ecol Evol 20:136-142.

Pometti CL, Cialdella AM, Vilardi JC and Saidman BO (2007) Morphometric analysis of varieties of Acacia caven: (Leguminosae, Mimosoideae): Taxonomic inferences in the context of other Argentinean species. Plant Syst Evol 264:239249.

Pometti CL, Vilardi JC, Cialdella AM and Saidman BO (2010) Genetic diversity among the six varieties of Acacia caven (Leguminosae, Mimosoideae) evaluated at molecular and phenotypic levels. Plant Syst Evol 284:187-199.

Pometti CL, Bessega CF, Vilardi JC and Saidman BO (2012) Landscape genetic structure of natural populations of Acacia caven in Argentina. Tree Genet Genomes 8:911-924.

Pritchard JK, Stephens M and Donnelly P (2000) Inference of population structure using multilocus genotype data. Genetics 155:945-959.

R Development Core Team (2011) R: A language and environment for statistical computing. R Foundation for Statistical Computing, Vienna, Austria.

Tishkoff S, Reed F, Friedlaender F, Ehret C, Ranciaro A, Froment A, Hirbo J, Awomoyi A, Bodo JM, Doumbo O, et al. (2009) The genetic structure and history of Africans and African Americans. Science 324:1035-1044.

Vos R, Hogers R, Bleeker M, Reijans M, Lee T, Hornes M, Frijters A, Pot J, Peleman J and Kuiper M (1995) AFLP: A new technique for DNA fingerprinting. Nucleic Acids Research 23:4407-4414.

\section{Internet Resources}

Jombart T (2012) A tutorial for Discriminant Analysis of Principal Components (DAPC) using adegenet 1.3-4. http://cran.r-project.org/web/packages/adegenet/vignettes/adegenet-dapc.pdf (October 23, 2012).

Pritchard JK, Wen X and Falush D (2009) STRUCTURE ver. 2.3. University of Chicago, Chicago, USA. http://pritch.bsd.uchicago.edu/ (December 29, 2010).

Pritchard JK, Wen X and Falush D (2010) Documentation for structure $\quad$ software: $\quad$ Version 2.3. http://pritch.bsd.uchicago.edu/structure.html (December $29,2010)$

\section{Associate Editor: Dario Grattapaglia}

All the content of the journal, except where otherwise noted, is licensed under a Creative Commons License CC BY-NC. 